myxomas), and to manage a possibly associated valve disease. $^{2}$

\section{References}

1. Reyen K. Cardiac myxomas. N Engl J Med. 1995;333:1610-7.
2. Davoli G, Muzzi L, Lucchese G, Uricchio N, Chiavarelli M. Large left atrial myxoma with severe mitral valve regurgitation. The inverted $\mathrm{T}$-shaped biatrial incision revisited. Tex Heart Inst J. 2006;33:51-3.

3. Feuchtner G, Mueller S, Bonatti J, Friedrich G, zur Nedden D, Smekal A. Prolapsing atrial myxoma. Dynamic visualization with multislice computed tomography. Circulation. 2004;109:e165-6.

\title{
An unusual case of a strangulated lung hernia after an Ivor Lewis esophagectomy
}

\author{
Rony Atoui, MD, MSc, Lawrence Lee, and Lorenzo Ferri, MD, PhD, Montreal, Quebec, Canada
}

Intercostal lung herniation is a rare condition that most commonly occurs after a traumatic event or through a congenital defect. We report an unusual case of a right lung hernia after an Ivor Lewis esophagectomy that was found to be strangulated and necrotic, requiring a wedge resection.

\section{CLINICAL SUMMARY}

A 30-year-old morbidly obese man with distal esophageal adenocarcinoma underwent an uneventful Ivor Lewis esophagectomy and thereafter had 3 cycles of docetaxel (Taxotere), cisplatin, and 5-flourouracil chemotherapy. An en-bloc resection with 2-field lymphadenectomy was performed through a laparotomy and right thoracotomy, and a hand-sewn esophagogastric anastomosis was generated cephalad to the azygous vein. The ribs were reapproximated with 4 interrupted figure eight, no. 1, nonlooped polydioxanone sutures. Postoperatively, the patient actively and enthusiastically participated in chest physiotherapy, including early ambulation and forced coughing. On postoperative day 8 , he had intermittent low-grade fever; however, a barium swallow and a chest computed tomographic (CT) scan did not reveal an anastomotic leak or mediastinal abscess. On postoperative day 11 , he experienced a sudden onset of right chest pain and dyspnea after an episode of vigorous coughing. Evaluation of the pleuritic pain revealed a localized tender bulge measuring $10 \times 5 \mathrm{~cm}$ at the level of the thoracotomy incision associated with massive subcu-

\footnotetext{
From the Division of Thoracic Surgery, Department of Surgery, McGill University Health Center, Montreal, Quebec, Canada.

Received for publication April 6, 2008; accepted for publication April 13, 2008; available ahead of print Aug 26, 2008.

Address for reprints: Lorenzo Ferri, MD, PhD, Division of Thoracic Surgery, McGill University Health Center, 1650 Cedar Ave, Montreal, Quebec, H3G 1A4, Canada (E-mail: lorenzo.ferri@muhc.mcgill.ca).

J Thorac Cardiovasc Surg 2009;138:495-6

$0022-5223 / \$ 36.00$

Copyright (c) 2009 by The American Association for Thoracic Surgery doi:10.1016/j.jtcvs.2008.04.026
}

taneous emphysema involving the right chest and neck. A CT scan of the chest revealed a large right pneumothorax associated with a herniation of a segment of the right lung parenchyma (Figure 1). On review of the previous CT scan, an uncomplicated minor lung hernia was evident.

The patient was brought to the operating theater after inserting a thoracostomy tube to relieve the pneumothorax. On entering the thoracic cavity, we confirmed the presence of a strangulated and necrotic right middle lobe hernia entrapped between the surrounding ribs (Figure 2). A wedge resection of this segment was performed, and the ribs were reapproximated with no. 1 looped polydioxanone sutures.

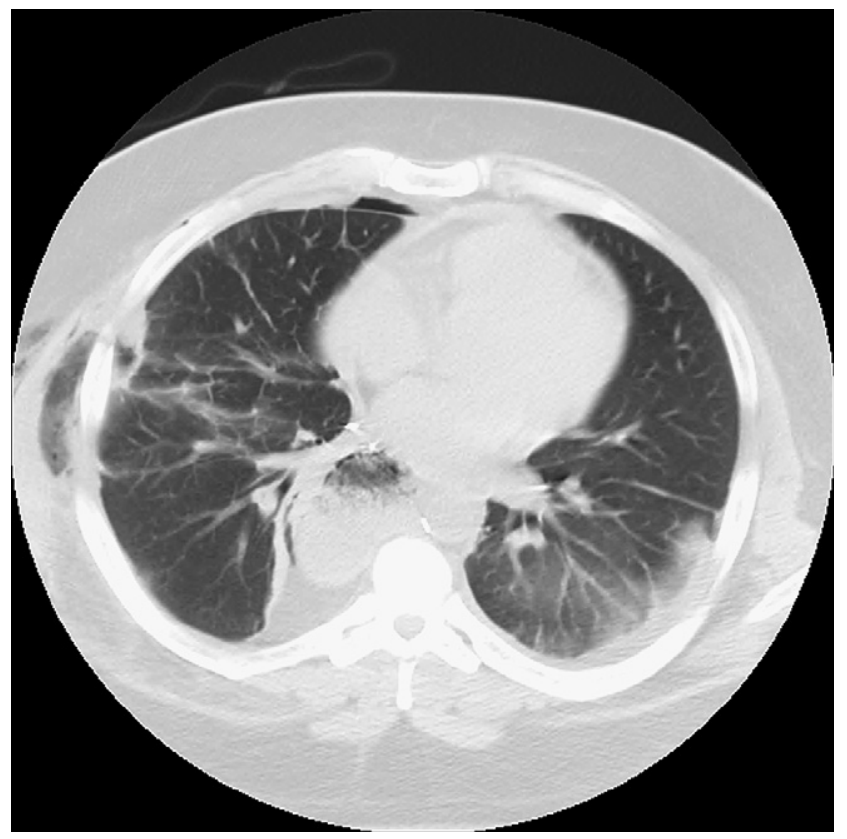

FIGURE 1. Preoperative computed tomographic scan of the chest demonstrating the herniation of a segment of the right lung associated with a pneumothorax. 

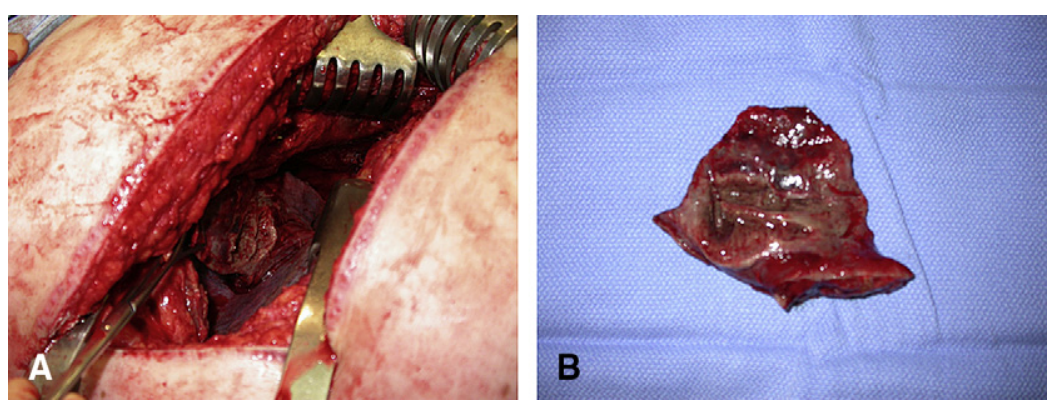

FIGURE 2. A, Intraoperative photograph showing the necrotic segment of the right middle lobe on exploration. B, Gross specimen of the excised segment of this right middle lobe. Note the infarcted area with hyperemic borders.

The patient tolerated the procedure well and was discharged home 3 days later.

\section{DISCUSSION}

Lung herniation is a distinctly rare entity, regardless of its location and cause, with approximately 300 cases reported in the literature since its initial description more than 5 centuries ago. ${ }^{1}$ They can be classified according to their cause (congenital or acquired) or anatomic location (cervical, thoracic, or diaphragmatic). ${ }^{2}$ Acquired hernias can be traumatic, spontaneous, or caused by neoplastic or inflammatory processes. Traumatic hernias have been reported as a result of falls, motor vehicle accidents, and penetrating chest trauma. ${ }^{3}$ Furthermore, lung hernias have been described after operative procedures, such as thoracotomy or thoracoscopic scars, or even at sites of previous chest tube insertions.

Patients at highest risk for lung hernia appear to be those with increased intrathoracic pressures, such as those with morbid obesity or end-stage chronic obstructive pulmonary disease. ${ }^{1}$ Other risk factors include tissue weakness or poor healing from malnutrition, steroids, diabetes, or other comorbidities. In our patient his body habitus, combined with the increased intrathoracic pressure caused by vigorous coughing, resulted in an acute lung herniation. Other contributing factors included his previous history of smoking and chemotherapy treatments.

Asymptomatic lung hernias might not require correction. However, surgical treatment is recommended in case of symptoms evoking incarceration, such as pain, or recurrent infections or for cosmetic reasons. ${ }^{3}$ Primary closure with pericostal fixation of the adjacent ribs is usually optimal, un- less a large defect necessitates use of muscle flaps or prosthetic material. ${ }^{1-3}$

In our patient his postoperative status, coupled with his morbid obesity, delayed the recognition and management of his lung hernia, which was found to be strangulated, necessitating a resection. Although repair of pulmonary lacerations of an incarcerated lung might be necessary, ${ }^{4}$ only rarely is it required to resect the incarcerated lung, as it was in our patient. In fact, strangulated lung herniation after an Ivor Lewis esophagectomy has not been previously documented. There is only 1 previously reported case of lung herniation after domino heart-lung transplantation that required a lobectomy because of infarction and necrosis. ${ }^{5}$

In summary, we describe the management of a patient who had a lung herniation after esophagectomy that was found to be strangulated, requiring a wedge resection. The possibility of a lung hernia should be considered in every patient who had a thoracotomy and who presents with dyspnea, a new fluctuant mass, and a pneumothorax. A higher index of suspicion is required in morbidly obese patients, especially after an episode of vigorous coughing. Because of symptoms and possible complications, early surgical repair is usually necessary and offers the best results with an excellent long-term prognosis. ${ }^{1,2}$

\section{References}

1. Allen GS, Fischer RP. Traumatic lung herniation. Ann Thorac Surg. 1997;63: 1455-6.

2. Weissberg D, Refaely Y. Hernia of the lung. Ann Thorac Surg. 2002;74:1963-6.

3. Arslanian A, Oliaro A, Donati G, Filosso PG. Posttraumatic pulmonary hernia. J Thorac Cardiovasc Surg. 2001;122:619-21.

4. Lang-Lazdunski L, Bonnet PM, Pons F, Bringuin L, Jancovici R. Traumatic extrathoracic lung herniation. Ann Thorac Surg. 2002;74:927-9.

5. Yonan NA, Egan J, Deiraniya AK, Rahman AN. Right lower lobe herniation after domino heart-lung transplantation. Ann Thorac Surg. 1995;59:1223-6. 